EPJ Web of Conferences 106, 07002 (2016)

DOI: $10.1051 /$ epjconf/201610607002

(C) Owned by the authors, published by EDP Sciences, 2016

\title{
Development of a Neutron Spectroscopic System Utilizing Compressed Sensing Measurements
}

\author{
Danilo Vargas $^{1}$, R. Cable Kurwitz ${ }^{2, \text { a }}$, Igor Carron ${ }^{3}$, and K. Russell DePriest ${ }^{4}$ \\ ${ }^{1}$ Undergraduate Student, Department of Nuclear Engineering, Texas A\&M University, College \\ Station, TX, USA \\ ${ }^{2}$ Director of Systems Engineering Initiative, Department of Nuclear Engineering, Texas A\&M \\ University, College Station, TX, USA \\ ${ }^{3}$ Manager and Editor, Nuit Blanche, Paris, France \\ ${ }^{4}$ Principal R\&D Scientist/Engineer, Applied Nuclear Technologies, Sandia National Laboratories, \\ Albuquerque, NM, USA
}

\begin{abstract}
A new approach to neutron detection capable of gathering spectroscopic information has been demonstrated. The approach relies on an asymmetrical arrangement of materials, geometry, and an ability to change the orientation of the detector with respect to the neutron field. Measurements are used to unfold the energy characteristics of the neutron field using a new theoretical framework of compressed sensing. Recent theoretical results show that the number of multiplexed samples can be lower than the full number of traditional samples while providing the ability to have some super-resolution. Furthermore, the solution approach does not require a priori information or inclusion of physics models. Utilizing the MCNP code, a number of candidate detector geometries and materials were modeled. Simulations were carried out for a number of neutron energies and distributions with preselected orientations for the detector. The resulting matrix (A) consists of $\mathbf{n}$ rows associated with orientation and $\mathbf{m}$ columns associated with energy and distribution where $\mathbf{n}<\mathbf{m}$. The library of known responses is used for new measurements $\mathbf{Y}(\mathbf{n} \times 1)$ and the solver is able to determine the system, $\mathbf{Y}=\mathbf{A x}$ where $\mathrm{x}$ is a sparse vector. Therefore, energy spectrum measurements are a combination of the energy distribution information of the identified elements of $\mathbf{A}$. This approach allows for determination of neutron spectroscopic information using a single detector system with analog multiplexing. The analog multiplexing allows the use of a compressed sensing solution similar to approaches used in other areas of imaging. A single detector assembly provides improved flexibility and is expected to reduce uncertainty associated with current neutron spectroscopy measurement.
\end{abstract}

\section{Introduction}

Neutron spectroscopy is an important topic of study and is essential to the characterization of neutron radiation fields. There are a wide variety of neutron spectrometers, most notably the Bonner Sphere

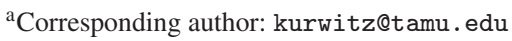

This is an Open Access article distributed under the terms of the Creative Commons Attribution License 2.0, which permits unrestricted use, distribution, and reproduction in any medium, provided the original work is properly cited. 
Spectrometer (BSS), which has been used for over four decades. The BSS is a set of spheres consisting of a thermal detector at the center of a ball of moderator material such as high-density polyethylene (HDPE). Incident neutron field measurements are taken individually for each different diameter sphere and the neutron energy spectrum is obtained using an unfolding technique on the set of detector responses.

Unfolding techniques or algorithms such as MAXED [1], GRAVEL [2], or FRUIT [3] unfolding codes have been developed and each has a unique set of characteristics regarding accuracy and applicability. For example, results from the MAXED algorithm are sensitive to an initial guess [4]. Although the aforementioned techniques for unfolding are still widely used, new approaches are being developed to provide better accuracy, which are less sensitive to initial conditions, and are less susceptible to error introduced in both the measurement and in the reconstruction. Examples of uncertainty sources include the inability to simultaneously collect detector responses, use of multiple detectors in each sphere, and cross section uncertainty used in the unfolding process.

Whereas the BSS technique relies on multiple detectors and moderator geometries tested sequentially, the proposed detector will be composed of a single detector with non-symmetric material design and will be modulated to change the incident neutron beam with respect to the detector. Ideally, each modulation would produce a unique response for a given energy level. The proposed detector draws inspiration from a previous study on the response of Bonner Sphere borehole orientation [5] and recent developments in the field of compressed sensing [6].

The main purpose of this study is to provide a proof-of-concept for both the measurement process and the energy spectrum reconstruction technique. MCNP6 [7] was used for simulating detector responses for various neutron energy spectrums. The calculational results are used as a calibration or detector response matrix (DRM), which is then used in evaluating new measurement queries. For our work, several neutron spectra, various energy biased beams, and multiple Maxwellian neutron energy spectra were used in the construction of the DRM. A MCNP simulation of the TRIGA research reactor at the Texas A\&M University Nuclear Science Center (NSC) was used as a query and reconstruction results are presented below.

\section{Approach}

The goal of the detector design was to produce a sparse vector where there would be little or no response except for a particular energy or range of energies. In order to achieve such a response, different materials and geometries were evaluated in order to determine appropriate materials and geometry. For our experiment, we chose to utilize two materials, a moderator and an absorber. More exotic material combinations were considered but not included for the proof-of-concept study.

A 1-D model of a $\mathrm{BF}_{3}$ detector was placed behind a wall of moderator and/or absorber material of varying thickness. Various neutron energy spectra were simulated and the effects were observed. Simulation results showed that the absorber material did not have to be of large thickness in order for it to have a desired effect on the response function. This result allowed absorber thickness reduction to less than $1 \mathrm{~mm}$ thick. The simulation was transitioned from a 1-D system into a 3-D system. The 1-D model of a changing moderator thickness was applied to the 3-D model as angular gradations in the spectrometer. The variation in moderator thickness made the response function dependent on the angle in which the spectrometer was positioned in an incident neutron beam.

\subsection{Evaluation of Calibration}

A first design of this conceptual spectrometer, as shown in Fig. 1, consists of a single $1.27 \mathrm{~cm} \mathrm{BF}_{3}$ cylindrical thermal detector coaxial with a larger cylinder split into quadrants with gradations of HDPE at both the outer diameter and surrounding the detector. A thin layer of boron lies along the inner 

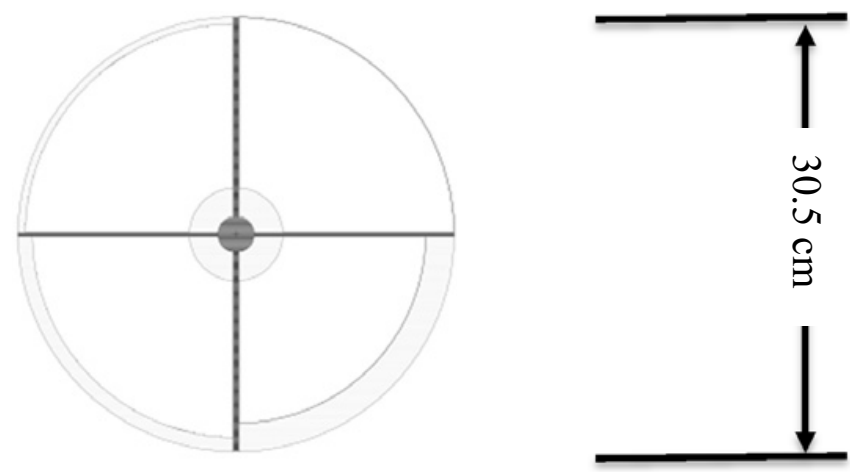

Figure 1. Top view of proposed detector schematic.

region of the HDPE gradations as an absorber, along with a thicker $0.41 \mathrm{~cm}$ cross-shaped barrier of boron splitting each quadrant. The spectrometer response is angular dependent since each of the four regions provided a different radial thickness of moderating material giving results similar to switching out different sized Bonner Spheres.

From Fig. 1, the center region is the $\mathrm{BF}_{3}$ detector, the region surrounding the detector and the gradations along the outer radius are the HDPE moderator, and the cross-shaped region and very thin layer along the inside of the moderator gradations are the boron absorber. The regions that are blank are voided regions containing no material. The moderator gradations are $0 \mathrm{~cm}, 0.5 \mathrm{~cm}, 1 \mathrm{~cm}$, and $2 \mathrm{~cm}$. The spectrometer is a right circular cylinder with a diameter and height equal to $30.5 \mathrm{~cm}$. The whole device was modeled in MCNP6.

\subsection{DRM Calculations by Monte Carlo Methods}

The DRM was calculated using MCNP6 simulations. A transformation was used to allow for clockwise movement of the device about the center z-axis. A Perl script was utilized to provide the transformation parameters needed to make 30 equally spaced angles between 0 and 360 degrees, to produce the various neutron spectra, and to produce a query of other neutron spectra for reconstruction. Two spectra from the TRIGA research reactor at Texas A\&M University were included as queries. A large number of spectra, 460, were generated using a number of different energy levels. Maxwellian spectra of different temperature (energy) and sources with uniform energy distributions over a limited range of energies were used as the dictionary to model detector response to known spectra. Neutron energies range from $10^{-11} \mathrm{MeV}$ to $100 \mathrm{MeV}$. In total, there were 462 different spectra with 30 angles for each spectrum, making a total 13860 MCNP6 input decks. The detector response for each variation was extracted in the form of a MCNP tally on the $(n, \alpha)$ reaction rate in the detector. The DRM was extracted using a Perl script and was converted into a MatLab matrix for use with an unfolding code.

\subsection{Reconstruction Approach}

Reconstruction of neutron spectra from a set of Bonner Sphere Responses is challenged due to the limited number of measurements. The limited measurement set results in an underdetermined system of equations, and the inversion of that full matrix system results in nonsensical values for neutron energy. Typically, Eq. (1) gives a detector response, $R_{i}$, as a function of the energy dependent microscopic cross 
section and energy dependent neutron fluence.

$$
R_{i}=\int_{E_{\min }}^{E_{\max }} \sigma_{i}(E) \phi(E) d E \quad \text { where } i=1,2, \ldots M .
$$

The relationship found in Eq. (1) is shown in matrix form as Eq. (2). Physically, is quite clear that $i \ll j$, so one must assume a physically appropriate form for a solution of $R$ in order to develop a solution.

$$
\bar{R}_{i}=\sigma_{i j} \bar{\phi}_{j}
$$

Compressed sensing (CS) is a recent technique designed to acquire signals in a multiplexed fashion. Examples of multiplexed operations are not uncommon in nuclear detectors. For instance, coded apertures cameras used for X-ray observations fall in that category, and there have been a number of recent applications of compressed sensing associated with these systems. Recent theoretical results show that the number of multiplexed samples can be lower than the full number of traditional samples but can also provide some super-resolution [8]. Furthermore, the solution approach does not require a priori information or inclusion of physics models.

Multiplexed signals are generally put together within the electronic chain of the signal processing, but in this case, multiplexing occurs in the neutron response of the detector design. Ideally, one sector of the detector will have a strong response to a specific energy level and almost no response to other neutron energies outside that region. Underlying requirements of our proposed detector are: sparsity, which pertains to the signals of interest, and incoherence, which pertains to the sensing modality [9]. Unlike most of the literature on compressive sensor development, we have shown sensors that perform multiplexing in analog fashion (i.e., a combination of materials and changing geometry).

Similar to other approaches, our method produces an underdetermined system of measurements but one that is sparse allowing the use of compressed sensing solvers. The detector response matrix (A) consists of $\mathbf{n}$ rows associated with orientation and $\mathbf{m}$ columns associated with different neutron spectra (B) where $\mathbf{n}<\mathbf{m}$. This DRM, A, of known responses is then used for new measurement queries $\mathbf{Y}(\mathbf{n} \times 1)$ and an appropriate compressed sensing (CS) solver is able to solve the system $\mathbf{Y}=\mathbf{A x}$ where $\mathrm{x}$ is a sparse vector. Compressed sensing algorithms utilize $1_{1}$-norm minimization to solve convex optimization problems subject to constraints. The sparse solution, $\mathrm{x}$, can then be applied to the known neutron spectra of $\mathbf{A}$ to determine the neutron energy spectrum, $\boldsymbol{\phi}$. Or stated mathematically, the combination of the energy distribution information is given by $\boldsymbol{\phi}=\mathbf{B x}$. This approach utilized a gradient projection for sparse reconstruction (GPSR) algorithm. A description of the algorithm can be found in the literature [10]. It is essentially a convex optimization code that utilizes a Barzilai-Borwein Gradient Projection approach [11].

\section{Results and Analysis}

A detector response matrix, $\mathbf{A}$, was produced that consisted of 30 rows corresponding to the angular detector response and 460 columns corresponding to Maxwellian and uniform distribution neutron energy spectrums. A detector response to a Maxwellian energy distribution, Y, was also produced. Using the GPSR algorithm, a reconstruction was carried out to solve the following relationship for x:

$$
Y=A x \text {. }
$$

The result for $\mathrm{x}$ is shown in Fig. 2. Note the sparsity of the solution with almost all index elements of $\mathrm{x}$ equal to zero.

The resulting predicted detector response is shown in Fig. 3 with a comparison to the actual response, Y. Better agreement between the actual and predicted can determined based on solution criteria to the 


\section{$15^{\text {th }}$ ISRD}

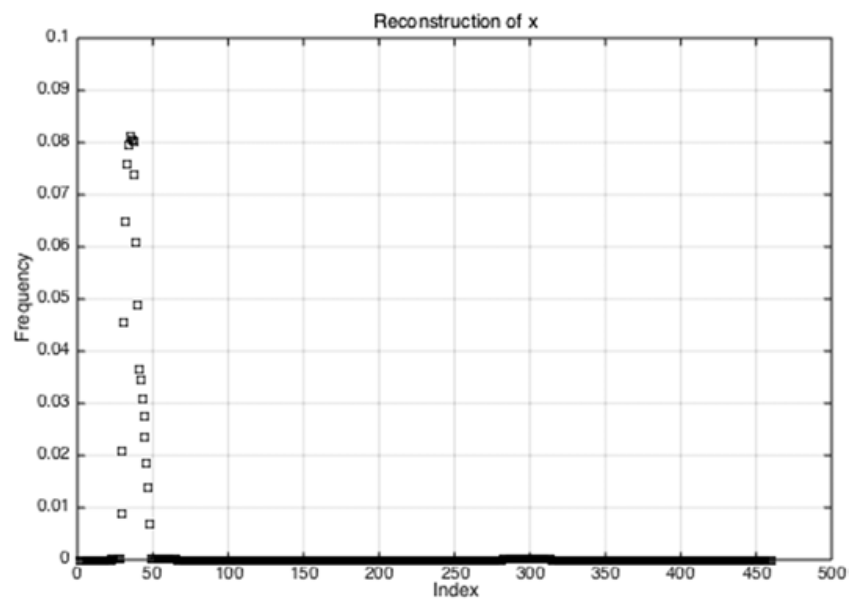

Figure 2. Reconstruction solution, $\mathrm{x}$, for $\mathbf{Y}=\mathbf{A x}$.

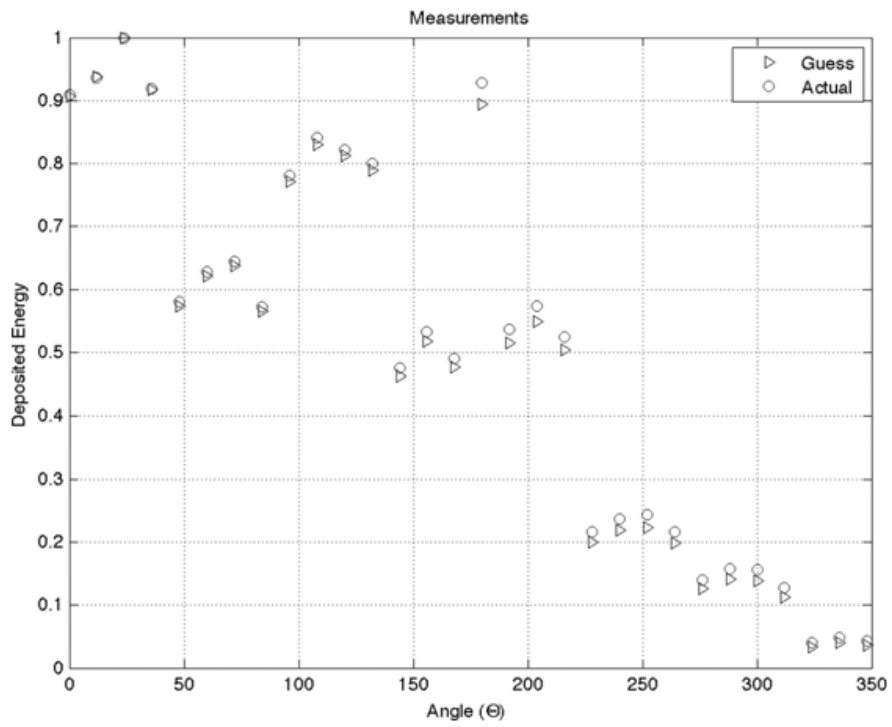

Figure 3. Comparison of Actual Detector Response and Response from Reconstruction.

reconstruction algorithm. MCNP is considered the actual response from the detector whereas the guess is generated using GPSR to solve the underdetermined system of equations $\mathbf{Y}=\mathbf{A x}$.

The predicted neutron spectrum, $\phi$, is calculated using Eq. (4) and takes the solution, $\mathrm{x}$, from Eq. (3) and multiplying by the matrix of neutron energy spectra, $\mathbf{B}$.

$$
\phi=B x .
$$

Figure 4 is a plot of the predicted neutron spectrum compared to the actual spectrum used in the query. The results indicate a proof-of-concept for a simple spectrum.

A more realistic neutron spectrum from the test reactor was evaluated as well. Using the neutron energy spectrum of the TRIGA test reactor at Texas A\&M University as the incident neutron flux, the detector response was determined. The same detector response matrix was used to determine the solution 


\section{EPJ Web of Conferences}

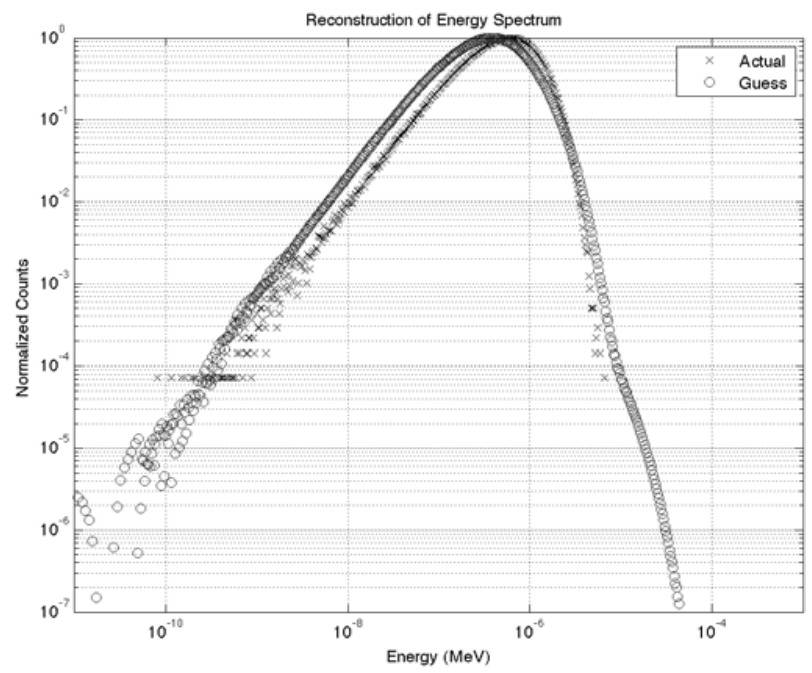

Figure 4. Comparison of Actual Neutron Spectrum and Predicted Neutron Spectrum.

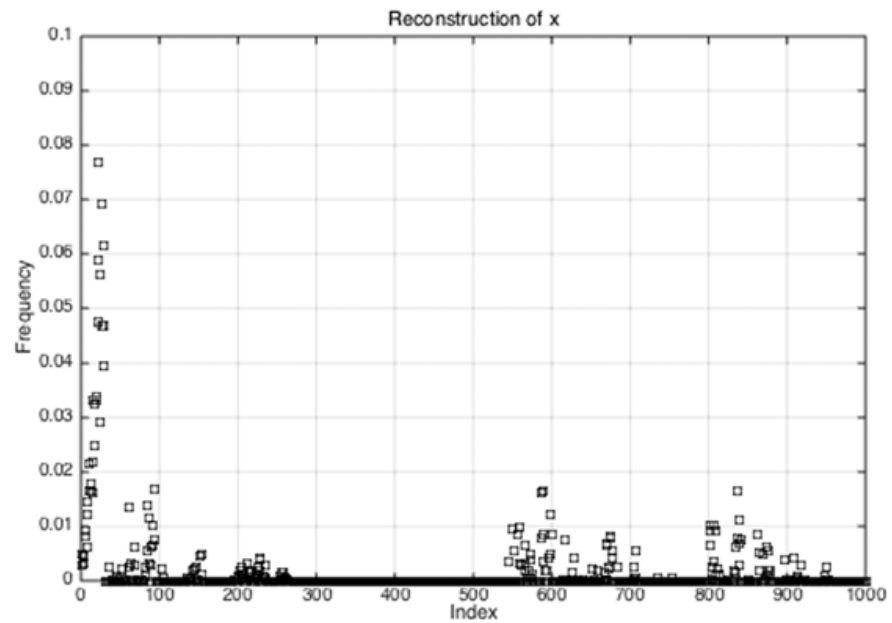

Figure 5. Reconstruction Solution, $x$, for TAMU-TRIGA Spectrum.

for $\mathrm{x}$. The detector response for the region directly outside of the TRIGA reactor core was simulated. This location includes both the thermal and fast peaks and will require multiple reconstruction elements or peaks in our solution for $\mathrm{x}$. The resulting plot of index elements is shown below in Fig. 5 and the reconstruction in Fig. 6.

The reconstruction captures the thermal and fast peaks relatively well but over predicts the epithermal region. Better reconstruction may be achieved by using a larger detector response matrix with a more diverse set of neutron spectrums that could include uniform and step distributions. In addition, changing detector materials could provide a different response in the epithermal regions resulting in greater sensitivity to this energy range. 


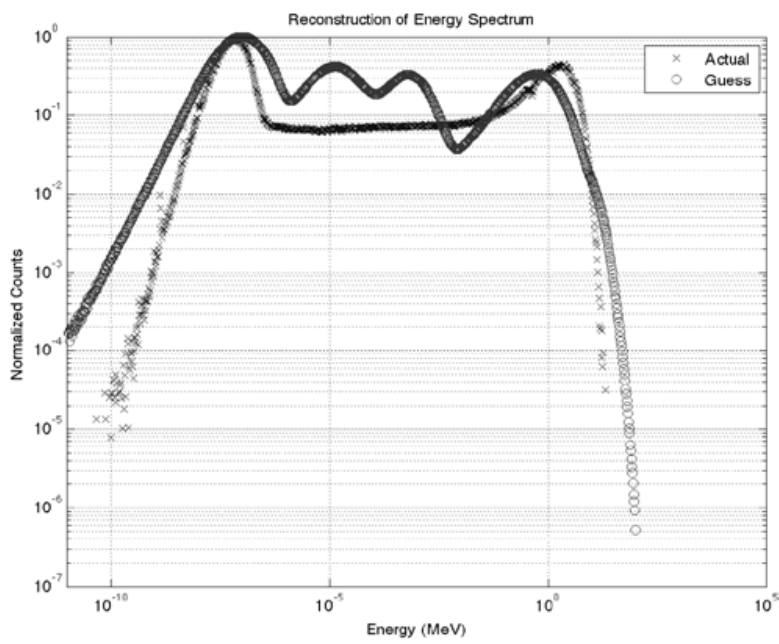

Figure 6. Comparison of Actual Neutron Spectrum and Predicted Neutron Spectrum for TAMU-TRIGA.

\section{Conclusions}

The approach presented in this paper allows for determination of neutron spectroscopic information using a single detector system with analog multiplexing. The use of detector orientation and material composition generates an angular dependent response that allows one to determine energy spectrum information. Analog multiplexing allows the use of a compressed sensing solution similar to approaches used in other areas of imaging. This approach provides a physically realistic solution without negative energies as opposed to least square solutions found in many reconstruction techniques [12]. A single detector assembly provides improved flexibility and is expected to reduce uncertainty associated with current neutron spectroscopy measurement.

The authors would like to thank the Nuclear Power Institute at Texas A\&M for providing support to the project as well as the Texas A\&M Nuclear Science Center for providing the MCNP model for the TRIGA reactor.

\section{References}

[1] Reginatto, M., Goldhagen, P., Neumann, S., "Spectrum unfolding, sensitivity analysis and propagation of uncertainties with the maximum entropy deconvolution code MAXED," Nuclear Instruments and Methods A 476, Issues 1-2, 242-246 (2002)

[2] Reginatto M., Wiegel B., Zimbal A. UMG code package version 3.2. PTB (2003)

[3] Bedogni, R., Domingo, C., Esposito, A. and Fernandez, F., "FRUIT: an operational tool for multi-sphere neutron spectrometry in workplaces," Nuclear Instruments and Methods A, 580, 1301-1309 (2007)

[4] Sundé, N., Andersson, E., et al. "Evaluation of spectral unfolding techniques for neutron spectroscopy," AIP Conference Proceedings, 988, No. 1 (2008)

[5] Brittingham, J., "The Effect of Bonner Sphere Borehole Orientation on Neutron Detector Response," Masters of Science Thesis, University of Tennessee (2010)

[6] Candès, E. J., Romberg J., and Tao, T., "Stable signal recovery from incomplete and inaccurate information," Communications on Pure and Applied Mathematics, 59, 1207-1233 (2005) 
[7] T. Goorley, M. James, T. Booth, F. Brown, J. Bull, L.J. Cox, J. Durkee, J. Elson, M. Fensin, R.A. Forster, J. Hendricks, H.G. Hughes, R. Johns, B. Kiedrowski, R. Martz, S. Mashnik, G. McKinney, D. Pelowitz, R. Prael, J. Sweezy, L. Waters, T. Wilcox, and T. Zukaitis, "Initial MCNP 6 Release Overview", LA-UR-11-07082, Los Alamos National Laboratory, also Nuclear Technology 180, pg 298-315 (Dec 2012)

[8] Donoho, D. L., "Superresolution via sparsity constraints," SIAM J. Math. Anal. 23, 1309-1331 (1992)

[9] Candès, E. J., and Wakin, M.B., "An introduction to compressive sampling," IEEE Signal Processing Magazine 21, no. 3, 21-30 (2008)

[10] Figueiredo, M.A.T., Nowak, R.D., and Wright, S.J., "Gradient projection for sparse reconstruction: application to compressed sensing and other inverse problems," IEEE Trans. Image Process 12, 906-916 (2003)

[11] Barzilai J. and Borwein, J., "Two point step size gradient methods," IMA Journal of Numerical Analysis 8, 141-148 (1988)

[12] Turlach, B., "On algorithms for solving least squares problems under an L1 penalty or an L1 constraint," Proceedings of the American Statistical Association; Statistical Computing Section, 2572-2577 (2005) 